\title{
Raman study of HIV TAR structural stability
}

\author{
Jan Vachoušek and Josef Štěpánek* \\ Faculty of Mathematics and Physics, Charles University, Prague, Czech Republic
}

\begin{abstract}
Structural characteristics of a central segment of the HIV RNA trans-activation response element (TAR) have been studied in aqueous solution. The investigated segment (TAR-14), comprising nucleotides 26-39 of the wild-type HIV genome (GAGC-CUGGGA-GCUC sequence), forms the TAR apical hairpin with a six-base loop and a stem of 4 base pairs. Raman spectra were recorded in a wide temperature range and the spectral series analyzed by means of factor analysis (FA) and a consequent fit to a thermodynamic model. The model was applied for temperatures above $20^{\circ} \mathrm{C}$ because of spectroscopic indication of possible intermolecular complexes at lower temperatures. FA results corresponded to a monomolecular reaction of the hairpin opening characterized by a melting temperature of $71^{\circ} \mathrm{C}$ and $\Delta G_{37}=-3.2 \mathrm{kcal} / \mathrm{mol}$. Besides the process of the hairpin opening, the FA revealed also substantial, approximately linear, temperature induced changes of the closed hairpin Raman spectrum. Spectral changes attributed to this effect indicated partial disordering of the A-type duplex geometry of the stem, opening of intraloop hydrogen bonds, weakened conformational regularity of the loop guanosines and release of the loop uracil.
\end{abstract}

Keywords: HIV, TAR, RNA, hairpin, Raman spectroscopy, factor analysis

\section{Introduction}

TAR (transactivation response) element is a structural motif located at the $5^{\prime}$-end of HIV genomic RNA. It plays very important role in the Tat-dependent transactivation of transcription that is essential for the HIV replication cycle (reviewed in [3]). In the HIV-1 type virus, this element usually consists with the first 59 nucleotides of the genome and is known to form an imperfect hairpin with a three-nucleotide bulge and a six-base loop. It serves as a scaffold for binding of viral Tat protein and cellular pTEFb factor (positive elongation factor b) consisting of CyclinT1 and CDK9 (cyclin dependent kinase 9) proteins [15].

Blocking the mechanism of Tat-dependent transactivation of transcription, the transcription efficiency of HIV virus is dramatically decreased. A possible way how to block the Tat-TAR interaction is occupation of the TAR binding site by a proper oligonucleotide aptamer. A few efficient RNA sequences have already been determined [6,8]. However, natural RNA aptamers are very rapidly degraded in living cells and chemically modified analogs of same sequences that are resistant to nuclease cleavage must be used instead for therapeutic purposes. Several of such chemically modified analogs of appropriate sequences have already been tested for their ability to bind tightly to their target sequence on TAR (reviewed in [17]). However, searching for suitable chemically modified analogs still remain a great challenge to research teams engaged in oligonucleotide therapy.

Knowledge of the TAR geometry and flexibility under different conditions is crucial to understand the mechanism of the TAR-aptamer interaction and to predict probable impacts of aptamer sugar-phosphate

\footnotetext{
*Corresponding author: Josef Štěpánek, Faculty of Mathematics and Physics, Charles University, Ke Karlovu 3, CZ-121 16 Prague 2, Czech Republic. Tel.: +420 221911 345; Fax: +420 224922 797; E-mail: stepjos@karlov.mff.cuni.cz.
} 


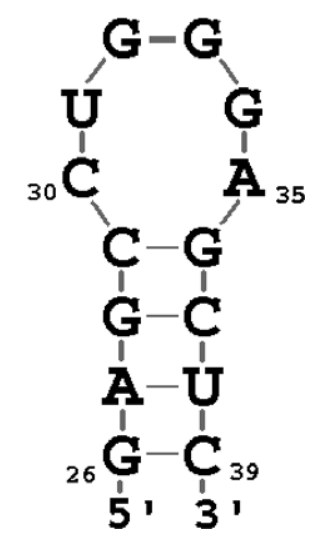

Fig. 1. Schematic draw of the TAR-14 hairpin.

backbone modifications on the aptamer binding ability and specificity. In our study we have concentrated on a 14-mer TAR segment (TAR-14) covering nucleotides 26-39 of the HIV-1 genome, i.e. the sequence G26-A27-G28-C29-C30-U31-G32-G33-G34-A35-G36-C37-U38-C39. TAR-14 represents the apical part of the TAR hairpin consisting of the six-base loop and the adjacent 4 base-pair stem (Fig. 1). This oligonucleotide has already been chosen for NMR structural study [11]. Although the obtained constrains did not lead to unambiguous loop structure, the authors obtained numerous indications about structural properties. Large proton-proton coupling interpreted as dynamic equilibrium between $3^{\prime}$-endo and $2^{\prime}$-endo sugar puckering was revealed not only for nucleosides in the loop, C30, G33 and G34, but also for G26 and C39 in the stem. This was interpreted as a sign of high flexibility of the structure. Three signals from imino-protons, which indicate shielding of the protons from direct contact with water, very probably because of their incorporation in interbase hydrogen bonds, were also detected for nucleosides in the loop. This result seems to be supported by a mutation study of UV detected stability in [12], where the authors concluded that the TAR apical loop is stabilized by a cross-loop base pair between C30 and G34.

In this work we present results achieved by a combination of Raman spectroscopy, advantageous because of its good applicability to biomolecular aqueous solutions at various conditions and high informational content in comparison with methods of electron optical spectroscopy, and a robust factor analysis providing clear insight into all tendencies in spectral variations.

\section{Experimental procedure}

The TAR-14 RNA oligonucleotide of $5^{\prime}$-r(GAGC-CUGGGA-GCUC)-3' sequence was synthesized and PAGE purified by VBC-Genomics (Austria). For Raman measurements, the oligonucleotide was dissolved in cacodylate buffer ( $20 \mathrm{mM}$ sodium cacodylate, $140 \mathrm{mM} \mathrm{KCl}, 20 \mathrm{mM} \mathrm{NaCl}, 3 \mathrm{mM} \mathrm{MgCl}{ }_{2}$, $\mathrm{pH}$ set to 6.5) so that the final oligonucleotide concentration was $1.4 \mathrm{mM}$.

Raman spectra were recorded from the sample placed in closed and temperature stabilized glass microcell of about $12 \mu \mathrm{l}$ total volume. The measurements were performed in a temperature range of $10-90^{\circ} \mathrm{C}$. The sample temperature was gradually increased with $3^{\circ} \mathrm{C}$ steps; 15 min equilibration period was hold prior to each measurement. Raman scattering was excited with the $514.5 \mathrm{~nm}$ line of $\mathrm{Ar}^{+}$ laser (Innova 300), radiant power at the sample was about $350 \mathrm{~mW}$. Raman scattering was collected in a 90-degree geometry and its spectrum, after a supernotch filtering of elastically scattered light, was 
recorded by a Spex 270M spectrograph (Jobin-Yvon) with a single 1800 grooves/mm grating and a liquid nitrogen cooled CCD $1340 \times 100$ (Princeton Instruments) detector. Total accumulation time was 15 min. Spectrum of calibration neon lamp was recorded after each Raman spectrum measurement and the data corrected for a possible subtle drifts of the wavenumber scale by using a homemade computer code.

Background correction was performed by subtracting optimal linear combination of ten spectral profiles comprising four analytical functions of arbitrary cubic polynomial, two profiles representing Raman spectrum of water and its temperature induced change, two profiles representing the same characteristics of cacodylate buffer, and at last two profiles given by the optical signal from the glass wall of the microcell and its temperature dependence. Background corrected spectra were further processed by factor analysis as described below.

\section{Results and discussion}

Obtained series of Raman spectra (Fig. 2) displays numerous temperature induced spectral changes occurring in various temperature regions. This Raman pattern seems to be typical for RNA hairpins,

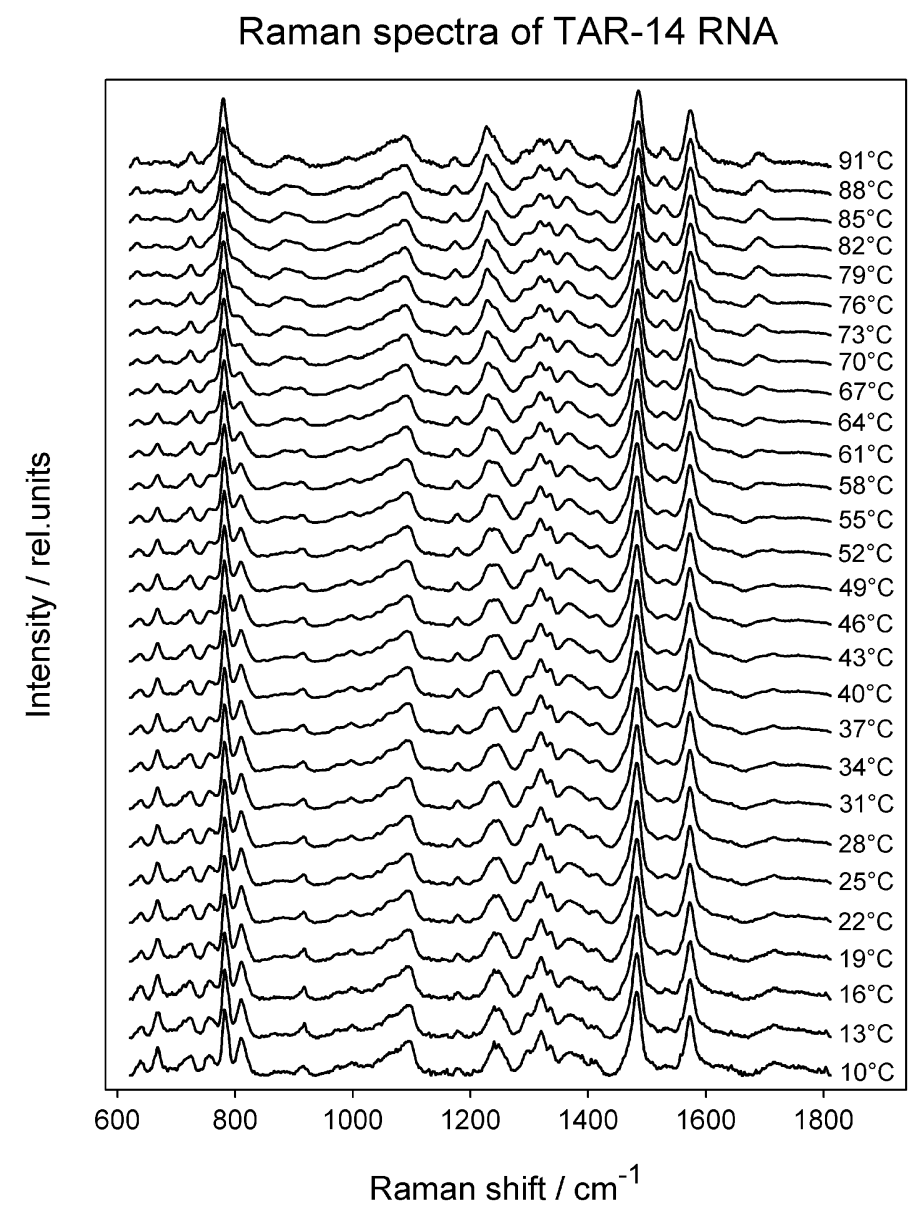

Fig. 2. Solution Raman spectra (background corrected) of TAR-14 hairpin forming RNA oligonucleotide at various temperatures. 
being observed for tetraloops and other hairpin types by several authors $[1,2,4,16]$. Inhomogeneity of the temperature effect was often revealed via different temperature profiles of particular Raman peak magnitudes. In our approach, application of factor analysis enables us to resolve all different tendencies in the induced spectral variations and to extract a complete set of attributed spectral features for each particular tendency.

We employed a singular value decomposition (SVD) algorithm that decomposes a set of $N$ spectra $Y_{i}(\tilde{\nu}), i=1,2, \ldots, N$, into a set of independent (but artificial) normalized spectral components

$$
U_{j}(\tilde{\nu}), \quad j=1,2, \ldots, N: \quad Y_{i}(\tilde{\nu})=\sum_{j=1}^{M} V_{i j} W_{j} U_{j}(\tilde{\nu}),
$$

where the normalized coefficient $V_{i j}$ quotes relative portion of the $j$ th spectral component $U_{j}(\tilde{\nu})$ in the $i$ th original spectrum $Y_{i}(\tilde{\nu})$ and the singular number $W_{j}$ stands for the statistical weight of the $j$ th spectral component. As the spectral components are in descending order by singular values, usually a few first terms at the right side of the equation is sufficient to approximate the original spectral set within an experimental error. This value of $M$ is referred to as factor dimension.

Figure 3 shows SVD results when applied to the complete set of Raman spectra for temperatures from 10 to $91^{\circ} \mathrm{C}$. Besides the singular values and the $V$ coefficients from the first to the fourth spectral component it also shows a residual error, i.e. the error of the approximation when the value of $M$ is set to a particular number. Both the singular values and residual errors indicate that the spectral set contains three significant components. The singular values reveal though two more components that are statistically just at the edge of the noise level.

The $V_{1}$ coefficients are only weakly changed with temperature. Because the first FA component represents certain kind of an average spectral shape over the series, it is a sign that the overall Raman intensity is not strongly influenced by varied temperature. This means that there is reduced global hypochromic effect. Such observation is in accordance with other Raman and UV absorption measurements of temperature dependent overall Raman intensity or absorbance, respectively, of nucleic acid loops (e.g. [7, $14,16])$.

The $V_{2}$ coefficients that are a measure of the dominant change of spectral shapes in the series, exhibit clear sigmoidal temperature profile at temperatures above $50^{\circ} \mathrm{C}$ indicating temperature induced dissociation reaction - in our case it is obviously the opening of the hairpin. Bellow $50^{\circ} \mathrm{C}$, the $V_{2}$ coefficients do not, however, show any inclination to a constant low-temperature asymptote but possess almost linear and relatively steep temperature dependence. This temperature profile of the FA second coefficients has been observed for RNA tetraloop in [14] and interpreted as a single very wide sigmoidal curve. The situation is actually revealed by the $V_{3}$ coefficients related to the second dominant spectral change displayed in the third spectral component. We can see that the temperature dependence consists of two distinct regions with opposite slopes. The boundary temperature is just about $50^{\circ} \mathrm{C}$, i.e. at the temperature where one expects to see the beginning of the sigmoid in the $V_{2}$ profile. This means that the sample exhibits at least two types of temperature induced spectral changes. The sigmoid profile in the high temperature region can be attributed to the hairpin opening while the changes visible in the low temperature region seem to indicate temperature induced variation of an average structure of the closed hairpin, caused by its low rigidity.

We have also to take into account possible formation of intermolecular complexes that may occur at low temperatures [7]. Temperature induced dissociation of such complexes would though cause deviations from the linear temperature dependence. Careful investigation of the low temperature end of the $V_{2}$ 
TAR-14 RNA, SVD of Raman spectra all temperatures
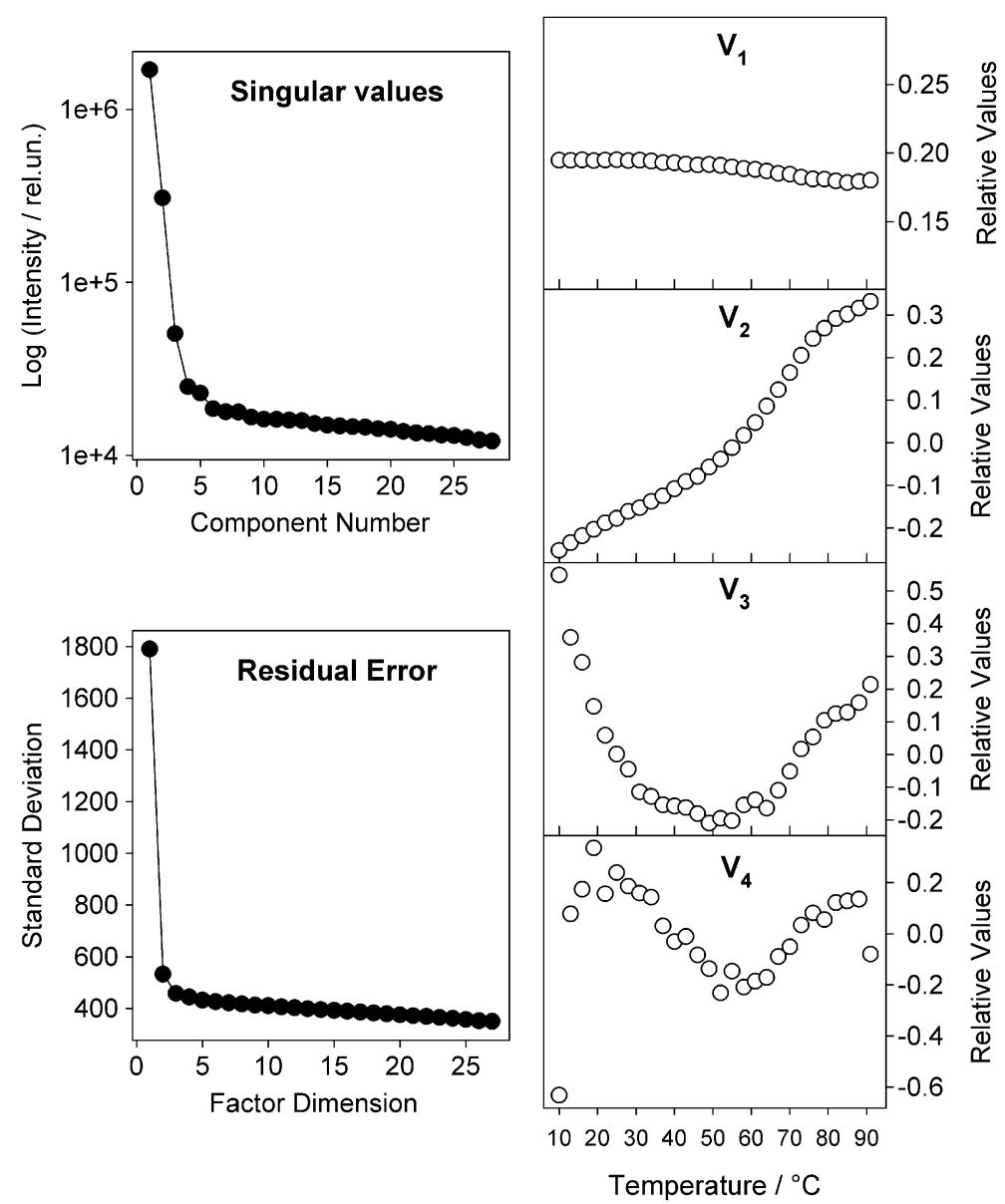

Fig. 3. Results of singular value decomposition applied to the spectral series from Fig. 2. Upper left: dependence of the singular values on the ordinal number of related spectral component. Lower left: dependence of the residual error on the number of spectral components taken into account. Right: temperature dependences of the coefficients indicating relative population of related spectral component in the measured spectrum.

profile reveals indeed very weak hook at temperatures bellow $20^{\circ} \mathrm{C}$. This correlates with a dramatic turn of the $V_{4}$ and $V_{5}$ profiles in this temperature region (only the $V_{4}$ coefficients are shown). On the other hand, expression of the different temperature behavior bellow $20^{\circ} \mathrm{C}$ just in the high-numbered components, the statistical weight of which is less than $1.5 \%$ of that of the first component, indicates clearly the weakness of the effect.

Nevertheless, the set of experimental spectra was in the further quantitative treatment reduced to temperatures above $20^{\circ} \mathrm{C}$ in order to avoid possible artifacts caused by minor presence of intermolecular complexes. The reduced spectral set was treated by SVD again; the results show corresponding reduction of dimensionality (Fig. 4). The temperature profiles of the $V_{1}, V_{2}$ and $V_{3}$ coefficients were then subjected to a global least square fit according to a model based on monomolecular opening reaction with an equilibrium constant fulfilling the Van't Hoff equation and on expected linear temper- 
TAR-14 RNA, SVD of Raman spectra temperatures above $20^{\circ} \mathrm{C}$
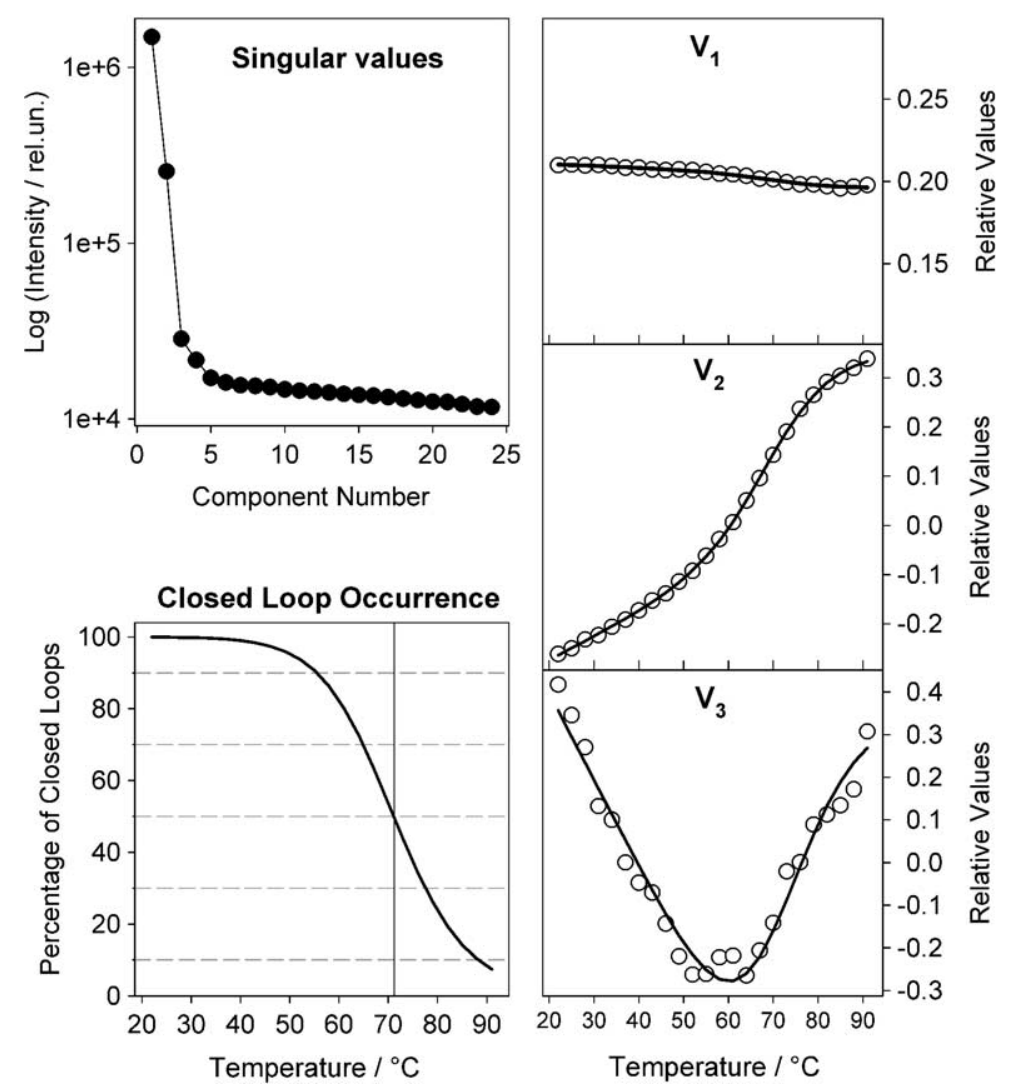

Fig. 4. Singular value decomposition applied to the spectral series from Fig. 2 but limited to temperatures higher than $20^{\circ} \mathrm{C}$. Results of the simultaneous fit of $V_{1}, V_{2}$ and $V_{3}$ coefficients to a thermodynamic model considering unimolecular opening reaction and linear temperature dependence of the closed hairpin Raman spectra are also described. Upper left: dependence of the singular values on the ordinal number of related spectral component. Right: temperature dependences of the coefficients indicating relative population of related spectral component in the measured spectrum. Experimental points (circles) and fitted theoretical curves (solid lines). Lower left: calculated temperature dependence of the closed loops percentage.

ature dependence of the closed hairpin spectrum. The fit therefore minimized sum of squared terms $V_{i j}-a_{j}^{\text {closed }}\left(1+\xi_{j}^{\text {closed }} T_{i}\right) C_{i}^{\text {closed }}-a_{j}^{\text {open }} C_{i}^{\text {open }}$ over all temperatures above $20^{\circ} \mathrm{C}$ and $j=1,2,3$. The terms corresponding to different spectral components were weighted by a square root of related singular value. Relative amounts $C_{i}^{\text {closed }}$ and $C_{i}^{\text {open }}$ of closed and opened hairpins were calculated according the Van't Hoff equation containing enthalpy and entropy differences connected with the hairpin formation as additional free parameters of the fit:

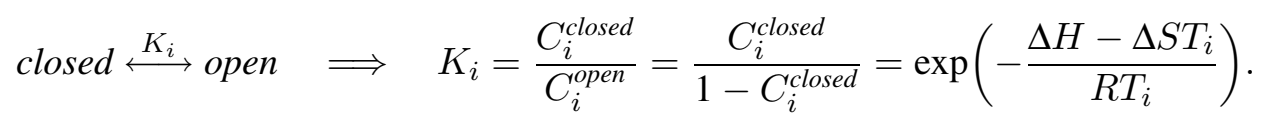

Results of the fit (shown in Fig. 4) demonstrate that the model perfectly suits the $V_{1}, V_{2}$ and $V_{3}$ profiles. The obtained $\Delta H$ and $\Delta S$ values give the melting (equilibrium) temperature of the hairpin opening 
$T_{\mathrm{m}}=71^{\circ} \mathrm{C}$ and $\Delta G_{37}=-3.2 \mathrm{kcal} / \mathrm{mol}$. Both values are close to those obtained in [11] from UV measurements at slightly different environmental conditions.

The results of the fit enabled simple isolation of Raman spectra corresponding to "pure" Raman spectra of closed and open hairpins. To demonstrate the temperature influence on Raman spectrum of closed hairpin and to distinguish these changes from the effect of the hairpin opening, we have brought out spectra of the open hairpin $Z^{\text {open }}(\tilde{\nu})$ spectrum and those of the closed hairpin corresponding to the lowest considered temperature $Z_{20}^{\text {closed }}(\tilde{\nu})$ and to the melting temperature $Z_{71}^{\text {closed }}(\tilde{\nu})$ according to

$$
\begin{aligned}
& Z^{\text {open }}(\tilde{\nu})=\sum_{j=1}^{3} a_{j}^{\text {open }} W_{j} U_{j}(\tilde{\nu}), \\
& Z_{20}^{\text {closed }}(\tilde{\nu})=\sum_{j=1}^{3} a_{j}^{\text {closed }}\left(1+\xi_{j}^{\text {closed }}(20+273.15)\right) W_{j} U_{j}(\tilde{\nu}), \\
& Z_{71}^{\text {closed }}(\tilde{\nu})=\sum_{j=1}^{3} a_{j}^{\text {closed }}\left(1+\xi_{j}^{\text {closed }}(71+273.15)\right) W_{j} U_{j}(\tilde{\nu}) .
\end{aligned}
$$

Obtained spectra and their mutual differences are shown in Fig. 5.

To interpret Raman spectra, we considered published results concerning spectra of RNA duplexes [5,9] and of RNA tetra-loop hairpins with various base compositions $[1,2,4,10,13,14]$. We have taken into account the published spectra and the interpretation given therein as well. Our assignment of individual Raman bands is listed in Table 1. The table also contains symbols of the close hairpin spectral changes induced by increased temperature and those caused by the hairpin opening.

Raman spectrum of the closed hairpin at $20^{\circ} \mathrm{C}$ exhibits clear indication of the dominant A-type structure of the stem by a relatively sharp and intensive marker band at $811 \mathrm{~cm}^{-1}$ and also a resolved band at $714 \mathrm{~cm}^{-1}$. The sharpness of the $811 \mathrm{~cm}^{-1}$ band of $\mathrm{O}-\mathrm{P}-\mathrm{O}$ stretching vibration contrasts, however, with the broadening of the $\mathrm{PO}_{2}{ }^{-}$stretching vibration band and the position of its maximum of $7 \mathrm{~cm}^{-1}$ downshifted from the value expected for A-form duplex. The shape, amplitude and position of this band are moreover surprisingly stable with increased temperature. The difference spectra reveal only minor nick indicating that a small portion of phosphate groups (not more than three from all) is at $20^{\circ} \mathrm{C}$ in the state corresponding to a regular A-form duplex. The O-P-O and the $\mathrm{PO}_{2}{ }^{-}$stretching vibrations seem therefore to be sensitive in a different way to the A-form duplex properties. As the former should be more sensitive to the backbone torsion angles, the later might be more sensitive to vibrational coupling with sugar molecules and thus reflect small instabilities of their puckering.

In fact, there are no direct signs for nucleosides in the stem to be in conformations different from the $3^{\prime}$-endo/anti (A-type) conformation. In opposite, the high intensity of $668 \mathrm{~cm}^{-1} \mathrm{rG}$ marker band indicates that not only the rG nucleotides in the stem but also at least one in the loop must be in $3^{\prime}$-endo/anti conformation. The weak $690 \mathrm{~cm}^{-1}$ band, on the other hand, indicates that some of the $\mathrm{rG}$ in the loop are in $2^{\prime}$-endo/anti state. Asymmetrical doublet at 1230 and $1250 \mathrm{~cm}^{-1}$ can be best explained by equal percentage of rU $3^{\prime}$-endo/anti and $2^{\prime}$-endo/anti conformations. As one rU is situated in the stem and one in the loop, it is natural to assign the $2^{\prime}$-endo/anti geometry to the rU in the loop.

There are no hypochromic bands except for the $\mathrm{rC}$ band at $1530 \mathrm{~cm}^{-1}$. Intensity increase of this band connected with the hairpin opening is so large that not only the cytosine in the stem but also the cytosine in the loop must stack with its neighbor base in the closed hairpin. 


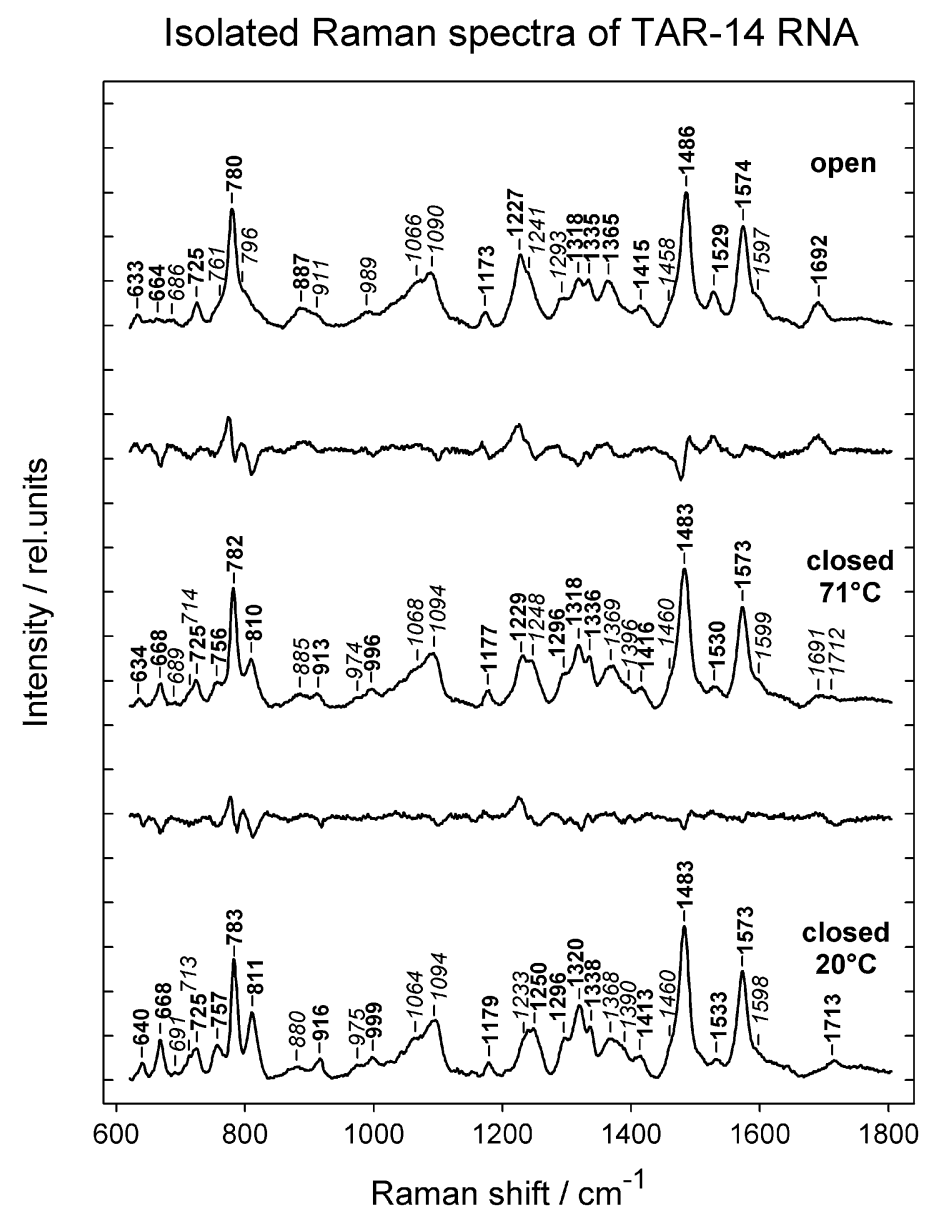

Fig. 5. Isolated Raman spectra of the closed TAR-14 hairpin at temperatures of $20^{\circ} \mathrm{C}$ and $71^{\circ} \mathrm{C}$ and spectrum of the open TAR-14 chain. Spectra of mutual differences are shown in the middle between relevant basic spectra.

Comparison of the closed hairpin spectra at $20^{\circ} \mathrm{C}$ and at $71^{\circ} \mathrm{C}$ reveals that increased temperature causes weakening of the stem A-form geometry: the $811 \mathrm{~cm}^{-1}$ band loses its intensity, the 782, 1483 and $1573 \mathrm{~cm}^{-1}$ bands are shifted closer to the positions in disordered structure. Another change, remarkable mainly in the difference spectrum, concerns transfer of Raman intensity from 1250 to $1227 \mathrm{~cm}^{-1}$. As the later vibrational frequency corresponds to $\mathrm{rU}$ in disordered structure, this change indicates release of the loop uridine (originally $2^{\prime}$-endo/anti) from stabilizing intraloop interactions. We can also notice moderate decrease of Raman scattering intensity at $1712 \mathrm{~cm}^{-1}$ and simultaneous increase at $1691 \mathrm{~cm}^{-1}$. This is a typical indication of the interbase disconnection. We can speculate that correlation of this spectral change with the uridine liberation means that the disconnection concerns the loop stabilizing internal bond.

Despite the temperature induced lost of structural rigidity, main structural characteristics are still kept in the closed hairpin as can be seen from the spectral changes caused by the hairpin opening. These changes include melting of the A-form geometry of the stem, lost of the regular rG $3^{\prime}$-endo/anti geometry $\left(668 \mathrm{~cm}^{-1}\right.$ marker), disconnection of all interbase H-bonds $\left(1712 \mathrm{~cm}^{-1}\right.$ marker), shift of 782, 1227, 1483 
Table 1

Raman bands of TAR-14 RNA loop and their temperature-induced changes

\begin{tabular}{|c|c|c|c|c|c|c|c|}
\hline Closed, $20^{\circ} \mathrm{C}^{\mathrm{a}}$ & Closed, $71^{\circ} \mathrm{C}^{\mathrm{a}}$ & Opened $^{\mathrm{a}}$ & E.T.I. ${ }^{\mathrm{b}, \mathrm{c}}$ & E.H.O., & Assignment ${ }^{\mathrm{d}, \mathrm{e}}$ & Significance $^{\mathrm{d}}$ & Refs \\
\hline $640 w$ & $634 w$ & $633 w$ & $\Downarrow \leftarrow$ & $\leftarrow$ & $\mathrm{rC}, \mathrm{rA}$ & & {$[5,9]$} \\
\hline $668 \mathrm{~m}$ & $668 w$ & $664 \mathrm{vw}$ & $\Downarrow$ & $\Downarrow$ & $\mathrm{rG}$ & rG $3^{\prime}$-endo/anti & {$[1]$} \\
\hline $691 v w$ & $689 v w$ & $686 v w$ & & & $\mathrm{rG}$ & rG $2^{\prime}$-endo/anti & {$[10]$} \\
\hline $713 v w, s h$ & $714 v w, s h$ & - & & $\Downarrow$ & $\mathrm{rG}$ or $\mathrm{rC}$ & $\begin{array}{c}\text { GC base-pair } \\
\text { in A-form duplex }\end{array}$ & {$[1,4,9]$} \\
\hline $725 \mathrm{~m}$ & $725 \mathrm{~m}$ & $725 \mathrm{~m}$ & - & - & A breath & & {$[5,9]$} \\
\hline $757 \mathrm{~m}$ & $756 w$ & $761 v w, s h$ & $\Downarrow$ & $\Downarrow$ & bk loop & & {$[1,13]$} \\
\hline $783 \mathrm{vs}$ & $782 \mathrm{vs}$ & $780 \mathrm{vs}$ & $\leftarrow$ & $\leftarrow$ & $\mathrm{C}+\mathrm{U}$ breath & at 784 in A-form duplex & {$[4,9]$} \\
\hline $811 s$ & $810 \mathrm{~m}$ & $796 w, s h, b r$ & $\Downarrow$ & $\Downarrow \leftarrow \rightarrow$ & $\mathrm{O}-\mathrm{P}-\mathrm{O}$ str & main A-form marker & {$[1,5,13]$} \\
\hline $880 v w, b r$ & $885 w, b r$ & 887 m,br & $\Uparrow$ & $\Uparrow$ & rib str & & [5] \\
\hline $916 \mathrm{w}$ & $913 w$ & $911 v w, s h$ & $\Downarrow$ & $\Downarrow \leftarrow$ & rib str & & [5] \\
\hline $975 v w$ & $974 v v w$ & - & $\Downarrow$ & $\Downarrow$ & N-type rib & & [1] \\
\hline $999 w$ & $996 w$ & $989 v w$ & $\leftarrow$ & $\leftarrow \Downarrow$ & N-type rib & at 1008 in A-form duplex & {$[1,9]$} \\
\hline $1064 m, s h, b r$ & $1068 m, s h, b r$ & $1066 \mathrm{~m}, \mathrm{sh}, \mathrm{br}$ & & & rib $\mathrm{C}-\mathrm{O}$ str & & [5] \\
\hline $1094 s, b r$ & $1094 s, b r$ & $1090 s, b r$ & $\Downarrow 1100$ & $\Downarrow 1100$ & $\mathrm{PO}_{2}{ }^{-}$sym. str & at 1001 in A-form duplex & {$[2,4,5]$} \\
\hline $1179 \mathrm{w}$ & $1177 \mathrm{w}$ & $1173 \mathrm{w}$ & $\leftarrow$ & $\leftarrow$ & rG (loop) & rG $3^{\prime}$-endo/syn marker & {$[1,2]$} \\
\hline 1233 m,as & $1229 \mathrm{~s}$ & $1227 \mathrm{~s}$ & $\Uparrow \leftarrow$ & $\Uparrow \leftarrow$ & rU ring str & rU $3^{\prime}$-endo/anti & [4] \\
\hline $1250 \mathrm{~s}$ & $1248 m, s h$ & $1241 \mathrm{~m}, \mathrm{sh}$ & $\Downarrow \leftarrow$ & $\Downarrow \leftarrow$ & $\mathrm{rU}, \mathrm{rC}$ ring str & $\begin{array}{l}\text { rU } 2^{\prime} \text {-endo/anti } \\
\text { rC } 3^{\prime} \text {-endo/anti }\end{array}$ & [4] \\
\hline $1296 \mathrm{~m}$ & $1296 \mathrm{~m}$ & 1293 w,as & $\Downarrow$ & $\leftarrow$ & $\mathrm{rC}$ & rC $3^{\prime}$-endo/anti & [4] \\
\hline $1320 \mathrm{~s}$ & $1318 \mathrm{~s}$ & $1318 \mathrm{~m}$ & $\Downarrow \leftarrow$ & $\Downarrow \leftarrow$ & $\mathrm{rG}$ & $\begin{array}{l}\text { at } 1315 \mathrm{rG} \mathrm{3} 3^{\prime} \text {-endo/anti } \\
\text { at } 1320 \mathrm{rG} \mathrm{3}{ }^{\prime} \text {-endo/syn }\end{array}$ & [13] \\
\hline $1338 \mathrm{~m}$ & $1336 \mathrm{~m}$ & $1335 \mathrm{~m}$ & $\leftarrow$ & $\leftarrow$ & $\mathrm{rA}$ & no interbase bond at $\mathrm{N} 7$ & [9] \\
\hline $1368 m, b r$ & $1369 \mathrm{~m}, \mathrm{br}$ & $1365 \mathrm{~m}$ & $\Uparrow 1365$ & $\Uparrow 1365$ & $\mathrm{rG}$ & at $1360 \mathrm{rG} 3^{\prime}$-endo/anti & {$[13]$} \\
\hline $1390 \mathrm{~m}, \mathrm{sh}$ & $1396 w, s h$ & - & $\Downarrow$ & $\Downarrow$ & $\mathrm{rU}$ & & [9] \\
\hline $1413 \mathrm{w}$ & $1416 \mathrm{w}$ & $1415 \mathrm{w}$ & & & rG & & {$[2,4,13]$} \\
\hline $1460 w, s h$ & $1460 w, s h$ & $1458 w, s h$ & & & rib $\mathrm{C}^{\prime} \mathrm{H}_{2}$ sciss & & [5] \\
\hline 1483 vs & 1483 vs & 1486 vs & $\mathrm{w} \rightarrow$ & $\rightarrow$ & $\mathrm{rG}$ & & {$[2,13]$} \\
\hline $1533 w$ & $1530 \mathrm{w}$ & $1529 \mathrm{~m}$ & $\leftarrow$ & $\Uparrow$ & $\mathrm{rC}$ & hypochromic & [2] \\
\hline 1573 vs & 1573 vs & 1574 vs & $\mathrm{w} \rightarrow$ & $\mathrm{w} \rightarrow$ & $\mathrm{rG}+\mathrm{rA}$ & $\begin{array}{c}\text { downshift } \\
\text { for interbase } \mathrm{H} \text {-bond }\end{array}$ & {$[2,9,13]$} \\
\hline $1598 v w, s h$ & $1599 v w, s h$ & $1597 w, s h$ & & $\Uparrow$ & $\mathrm{rC}$ & & {$[2,13]$} \\
\hline- & $1691 w, b r$ & $1692 \mathrm{~m}, \mathrm{br}$ & $\Uparrow$ & $\mathrm{s} \Uparrow$ & $\mathrm{C}=\mathrm{O}$ str & not interbase $\mathrm{H}$-bonded & {$[2,10]$} \\
\hline 1713 w,br & $1712 v w, b r$ & - & $\Downarrow$ & $\Downarrow$ & $\mathrm{rU}, \mathrm{rG} \mathrm{C}=\mathrm{O}$ & interbase $\mathrm{H}$-bonded & {$[2,4]$} \\
\hline
\end{tabular}

${ }^{\text {a }}$ Peak positions are in wavenumber units $\left(\mathrm{cm}^{-1}\right)$. Numbers in bold correspond to well resolved bands; precision of the peak position is $\pm 1 \mathrm{~cm}^{-1}$. Numbers in italics correspond to shoulders, very broad, asymmetrical or partly overlapped bands; precision of the peak position is about $\pm 3 \mathrm{~cm}^{-1}$. Added basic characteristics of Raman bands: $\mathrm{w}=\mathrm{weak}, \mathrm{m}=$ medium, $\mathrm{s}=$ strong, $\mathrm{vs}=$ very strong, $\mathrm{vw}=$ very weak, $\mathrm{sh}=$ shoulder, $\mathrm{br}=$ broad, as $=$ asymmetric. ${ }^{\mathrm{b}}$ Symbols: $\Uparrow$ intensity increase, $\Downarrow$ intensity decrease, $\rightarrow$ upshift of vibrational frequency, $\leftarrow$ downshift of vibrational frequency, $\leftarrow \rightarrow$ broadening. If the intensity increase or decrease in the difference spectrum is not pronounced exactly at the frequency corresponding to the basic Raman band position, the position of the peak or nick in the difference spectrum is indicated. Letter " $w$ " prior to a symbol means weak spectral change. ${ }^{c}$ E.T.I. $=$ Effect of temperature increase; E.H.O. = Effect of the hairpin opening. d Abbreviations: A, C, G, U = adenine, cytosine, guanine, uracil; rA, rC, rG, rU = ribonucleotide containing given nucleobase; $\mathrm{bk}=$ backbone; rib $=$ ribose. ${ }^{\mathrm{e}}$ Abbreviations for vibrational modes: $\mathrm{str}=$ stretching, breath $=$ breathing, sciss $=$ scissoring, sym $=$ symmetric. 
and $1573 \mathrm{~cm}^{-1}$ bands to the positions corresponding to disordered structure, and loss of $\mathrm{rC}$ stacking (increased intensity of $1530 \mathrm{~cm}^{-1}$ band).

\section{Conclusions}

Our Raman study has proved that the hairpin forming TAR segment does not create any intermolecular complexes at temperatures above $20^{\circ} \mathrm{C}$ even at the used $1.4 \mathrm{mM}$ concentration. Temperature induced hairpin opening is characterized by a melting temperature of $71^{\circ} \mathrm{C}$. Factor analysis of temperature dependent Raman spectra has though revealed that the average structure of the closed hairpin is remarkably temperature dependent. This finding indicates low rigidity of the hairpin geometry. Temperature increase leads to a partial disordering of the A-type duplex geometry of the stem and to a loss of the six-member loop geometry, consisting in opening of intraloop hydrogen bonds, weakened regularity of the loop guanidine conformations, and release of the loop uracil.

The results not only enrich a general knowledge of the TAR RNA structural properties and their temperature dependence, but also represent solid methodological base for future studies of interactions between TAR and various aptamers.

\section{Acknowledgement}

This work was supported by the Czech Science Foundation (project No. 203/05/0827), the Grant Agency of Charles University (project No. 1475/07) and the Czech Ministry of Education (project MSM 0021620835).

\section{References}

[1] M. Abdelkafi, N. Leulliot, V. Baumruk, L. Bednarova, P.Y. Turpin, A. Namane, C. Gouyette, T. Huynh-Dinh and M. Ghomi, Structural features of the UCCG and UGCG tetraloops in very short hairpins as evidenced by optical spectroscopy, Biochemistry 37 (1998), 7878-7884.

[2] M. Abdelkafi, N. Leulliot, M. Ghomi, C. Herve du Penhoat, A. Namane, C. Gouyette, T. Huynh-Dinh, V. Baumruk and P.Y. Turpin, UNCG tetraloops in short oligoribonucleotides reveal high thermodynamic stability and unusual structural properties in aqueous phase as confirmed by optical and NMR spectroscopies, J. Mol. Struct. 408/409 (1997), 241-245.

[3] M. Barboric and B.M. Peterlin, A new paradigm in eukaryotic biology: HIV Tat and the control of transcriptional elongation, PLoS Biology 3(2) (2005), e76.

[4] V. Baumruk, C. Gouyette, T. Huynh-Dinh, J.-S. Sun and M. Ghomi, Comparison between CUUG and UUCG tetraloops: thermodynamic stability and structural features analyzed by UV and vibrational spectroscopy, NAR 29(19) (2001), 40894096.

[5] J.M. Benevides, M. Tsuboi, J.K. Bamford and G.J. Thomas, Polarized Raman spectroscopy of double-stranded RNA from bacteriophage phi6: local Raman tensors of base and backbone vibrations, Biophys. J. 72 (1997), 2748-2762.

[6] F. Darfeuille, D. Sekkai, E. Dausse, G. Kolb, L. Yurchenko, C. Boiziau and J.J. Toulme, Driving in vitro selection of AntiHIV-1 TAR aptamers by magnesium concentration and temperature, Comb. Chem. High Throughput Screen. 5 (2002), 313-325.

[7] T.M. Davis, L. McFail-Isom, E. Keane and L.D. Williams, Melting of a DNA hairpin without hyperchromism, Biochemistry 37 (1998), 6975-6978.

[8] F. Duconge and J.J. Toulme, In vitro selection identifies key determinants for loop-loop interactions: RNA aptamers selective for the TAR RNA element of HIV-1, RNA 5 (1999), 1605-1614.

[9] J. Hanus, J. Stepanek, P.Y. Turpin and J. Bok, Formation and stability of nucleotide complexes: Raman titration investigation, J. Mol. Struct. 480/481 (1999), 437-442.

[10] B. Hernandez, V. Baumruk, N. Leulliot, C. Gouyette, T. Huynh-Dinh and M. Ghomi, Thermodynamic and structural features of ultrastable DNA and RNA hairpins, J. Mol. Struct. 651-653 (2003), 67-74. 
[11] J.A. Jaeger and I. Tinoco, An NMR study of the HIV-1 TAR element hairpin, Biochemistry 32 (1993), 12522-12530.

[12] T. Kulinski, M. Olejniczak, H. Huthoff, L. Bielecki, K. Pachulska-Wieczorek, A.T. Das, B. Berkhout and R.W. Adamiak, The apical loop of the HIV-1 TAR RNA hairpin is stabilized by a cross-loop base pair, J. Biol. Chem. 278(40) (2003), 38892-38901.

[13] N. Leulliot, V. Baumruk, M. Abdelkafi, P.Y. Turpin, A. Namane, C. Gouyette, T. Huynh-Dinh and M. Ghomi, Unusual nucleotide conformations in GNRA and UNCG type tetraloop hairpins: evidence from Raman markers assignments, NAR 27(5) (1999), 1398-1404.

[14] N. Leulliot, V. Baumruk, C. Gouyette, T. Huynh-Dinh, P.Y. Turpin and M. Ghomi, Aqueous phase structural features of GNRA tetraloops formed in short hairpins as evidenced by UV absorption and Raman spectroscopy, Vibr. Spectr. 19 (1999), 335-340.

[15] B.M. Peterlin and D.H. Price, Controlling the elongation phase of transcription with P-TEFb, Mol. Cell 23 (2006), 297305.

[16] V. Reipa, G. Niaura and D.H. Atha, Conformational analysis of the telomerase RNA pseudoknot hairpin by Raman spectroscopy, RNA 13 (2007), 108-115.

[17] J.J. Toulme, F. Darfeuille, G. Kolb, S. Chabas and C. Staedel, Modulating viral gene expression by aptamers to RNA structures, Biol. Cell 95 (2003), 229-238. 


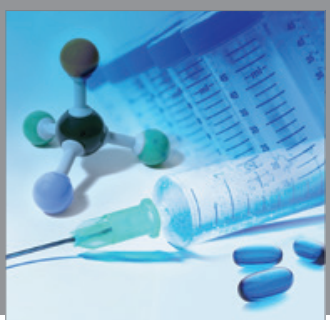

International Journal of

Medicinal Chemistry

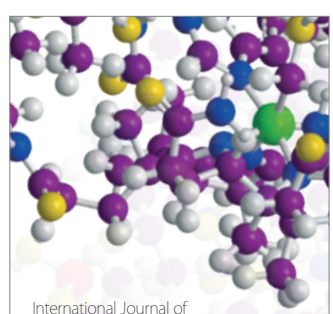

Carbohydrate Chemistry

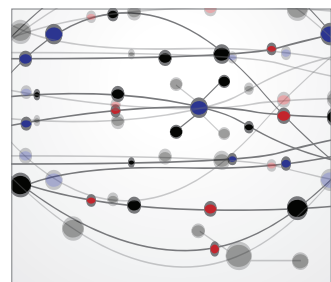

The Scientific World Journal
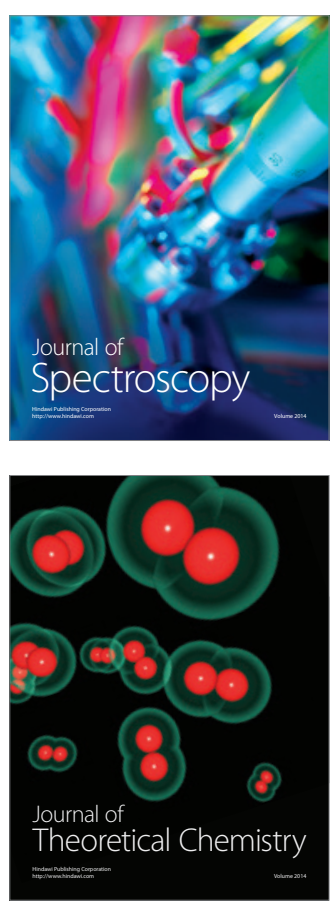
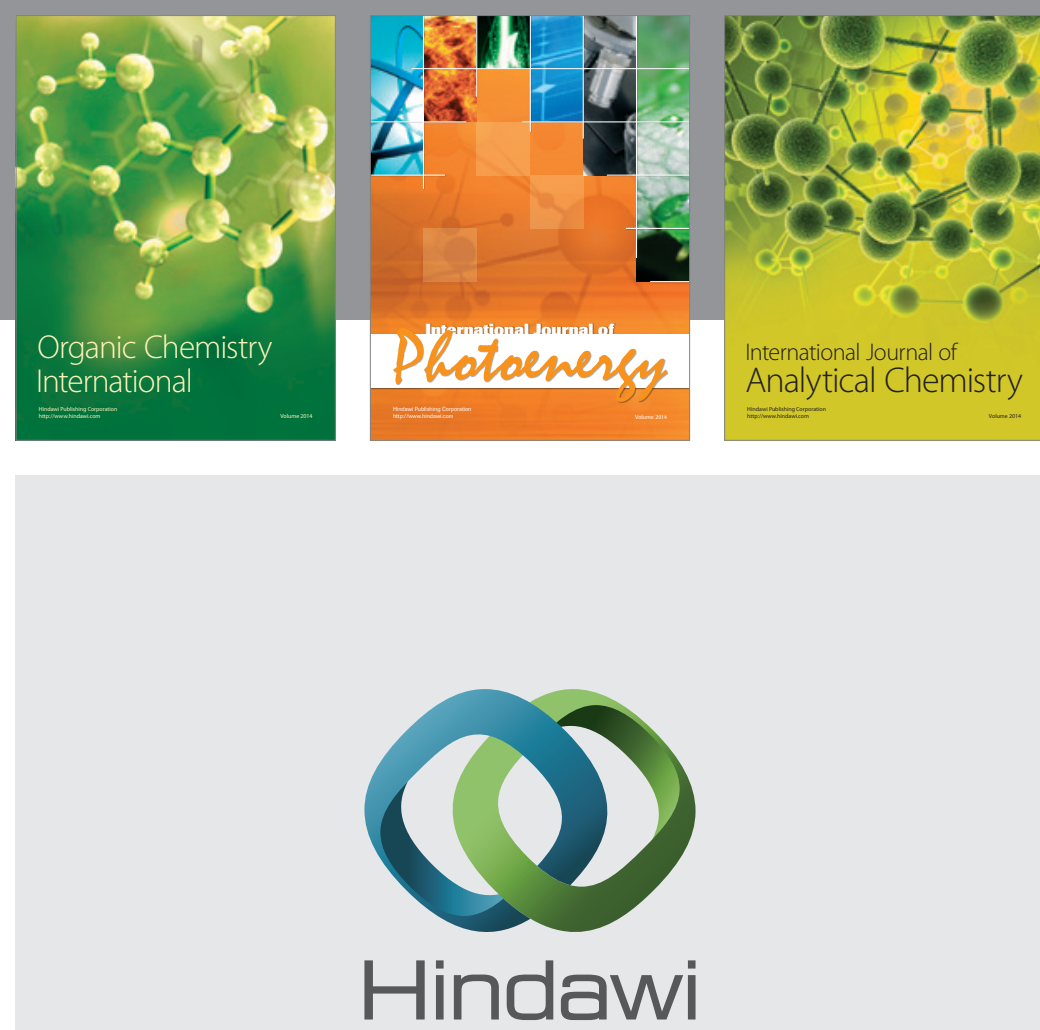

Submit your manuscripts at

http://www.hindawi.com
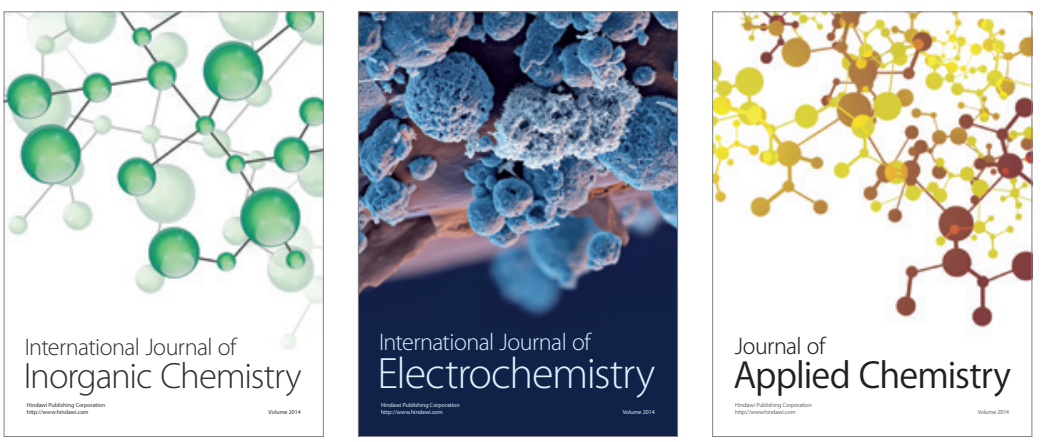

Journal of

Applied Chemistry
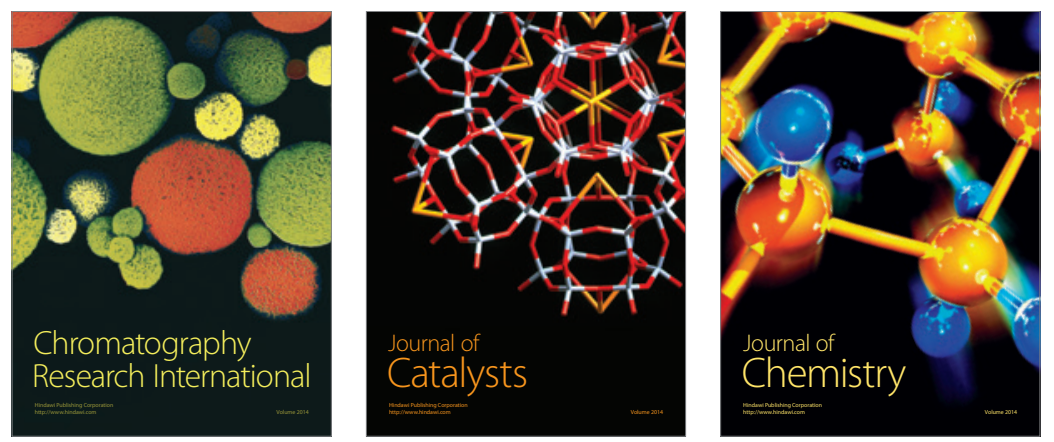
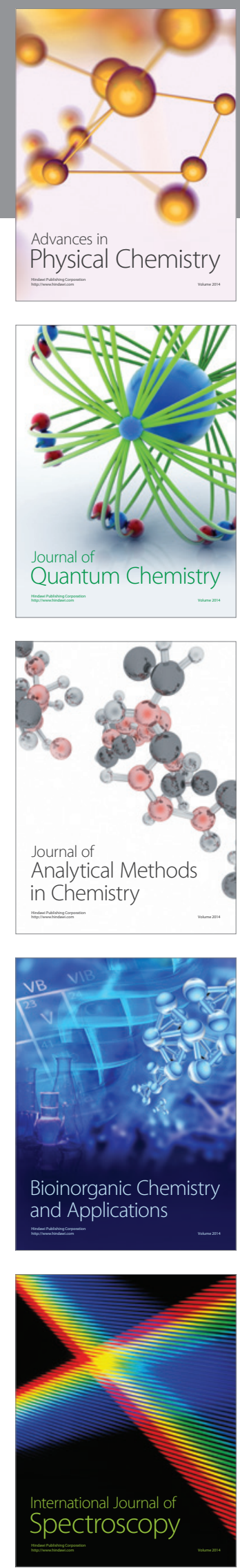